\title{
Detection of Internet Scam Using Logistic Regression
}

\author{
Mehrbod Sharifi \\ mehrbod@cs.cmu.edu
}

\author{
Eugene Fink \\ eugenefink@cmu.edu
}

\author{
Jaime G. Cabonell \\ jgc@cs.cmu.edu
}

Computer Science, Carnegie Mellon University, Pittsburgh, PA 15217

\begin{abstract}
Internet scam is fraudulent or intentionally misleading information posted on the web, usually with the intent of tricking people into sending money or disclosing sensitive information. We describe an application of logistic regression to detection of Internet scam. The developed system automatically collects 43 characteristic statistics of the websites from 11 online sources and computes the probability that a given website is malicious. We present an empirical evaluation of this system, which shows that its precision and recall are $98 \%$.
\end{abstract}

Keywords-Machine Learning, Web Security.

\section{INTRODUCTION}

Internet scam is a type of security threat in which a website makes false or intentionally misleading claims, usually with the purpose to trick its visitors into paying for fake product offers or providing sensitive identity information. Examples include fraudulent promises to help find work at home and cure various diseases. The detection of such scams is difficult because fraudulent sites usually use effective deception techniques that make them appear legitimate, such as fake testimonials and success stories, as well as genuine references to trusted sources, such as Wikipedia, specific scientific publications, and patents

A related well-studied problem is the detection of email spam, that is, unwanted bulk email. The subject matter of spam emails is a good indicator of types of scams. Some types of scams are almost entirely distributed through spam email and scammers often engage in spamvertising that is, sending emails with links to scam websites [Anderson et al., 2007]. The performance of the modern spam filters is quite impressive; the best systems in TREC 2007 spam filtering challenge had the AUC score over 99.99\% [Cormack, 2007a].

A more recent related problem is the emergence of web spam, also called spamdexing, which is "any deliberate human action that is meant to trigger an unjustifiably favorable relevance or importance for some web page, considering the page's true value" [Gyöngyi and Garcia-Molina, 2005]. That is, scammers try to bias the search results, using techniques such as keyword stuffing of web pages and creating a large number of links from other sites to spam pages. There are two broad types of approaches for detection of web spam, called content-based and link-based [Becchetti et al., 2008]. Currently, the content-based approaches tend to be more effective, and the underlying techniques are almost identical to those used for detecting email spam [Cormack 2007b; Cormack 2010]. Search engines have considerably improved in recent years to deal with web spam.

While web spam targets search mechanisms, web scam is aimed directly at the users, and the problem is still largely unaddressed. For legal reasons, search engines are reluctant to block scammers unless they have specific strong proof of fraudulent activity, such as confirmed instances of malware distribution. Additionally, scammers often use cloaking, that is, showing benign contents to search engines but different scam contents to visitors. They may frequently change their domain name, which makes it harder for search engines to judge the contents.

Currently, the most common approach to fighting Internet scam is blacklisting. Many public services such as hostsfile.net and smapcop.net, maintain a list of suspicious websites, compiled through user reports. The Web of Trust (mywot.com) includes several million users who rank websites on vendor reliability, trustworthiness, privacy, and child safety.

The main limitations of the blacklist approach are wellknown. First, a list may not include recently created or moved scam websites (false negatives). Second, it can mistakenly include legitimate websites because of inaccurate or intentionally biased reports (false positives). Scammers may intentionally bias blacklists in both directions.

We propose to reduce the omissions and biases in blacklists by integrating information from various heterogeneous sources, particularly focusing on the quantitative measurements that are hard to manipulate, such as the web traffic rank or the number of search engine results pointing to a website. We have applied a logistic regression algorithm to this set of automatically collected statistics. This machine learning approach is supervised, which means that we need a dataset of known scam and legitimate websites in order to train the system. We describe how we have created such labeled datasets after we provide a preliminary taxonomy of web scam types. Then we describe the core engine of our system, which collects necessary information for the scam detection algorithm. Finally, we present an empirical evaluation of the developed technique.

\section{COMMON TYPES OF INTERNET SCAM}

We are not aware of any exiting public datasets of Internet scam. To create our own dataset for supervised learning, we have started by studying the channels of scam distribution, and we have identified the following main channels. 
- Spam emails.

- User-generated contents of generally benign websites, such as blog comments and user reviews.

- Online advertisement: Ad networks with banners or textual ads; classified ads on Craigslist, Ebay, Amazon, and other similar websites.

- Outside Internet: Hard mail; TV and radio ads.

We have used these sources to create our datasets; in particular, we have manually analyzed 200 randomly selected spam emails to create this preliminary taxonomy of Internet scam types, which is probably not exhaustive:

1. Medical: Fake disease cure, longevity, weight loss, performance enhancer drugs.

2.Phishing: Pretending to be from a well known legitimate company, such as PayPal, and requesting a user action. The more recent variations include notifications of failed UPS and FedEx shipments, and gift cards from department stores.

3. Advance payout: Requests to make a payment in expectation of a large future gain, such as lottery prize or inheritance.

4. False deals: Fake offers of products at unusually steep discounts, such as cheap medications, insurance, and software.

5. Other: False promises of online degrees, work at home, dating, and other highly desirable opportunities.

\section{DATASETS}

We have created five labeled datasets of scam and legitimated websites, which are summarized in Table 1.

1. Scam queries: Certain search queries are especially likely to return a large number of scam websites. We have issued the following three queries to Google and recorded the top 500 results for each: "cancer treatments", "work at home", and "mortgage loans". After removing duplicates and all non-HTML links such as PDFs and videos, we have randomly picked 100 domain names for each query and submitted them to the Amazon Mechanical Turk for manual labeling as scam or non-scam. For each link, we have collected three human opinions. We have asked the Turk "workers" to review each website, decide whether it is scam or not, and indicate their level of confidence (highly confident, somewhat confident, or not confident). If all three people who labels a website provided the same opinion with high confidence, we have included the site into the dataset. If the human labelers disagreed about a website or indicated low confidence, we have not included it into the final set.

2. Web of Trust: To compile a set of popular links, we have collected the 200 most recent threads on the "Comments on websites" forum of Web of Trust (mywot.com). We have extracted all domain names mentioned in these discussion threads, thus obtaining 159 unique names. To increase diversity of this dataset, we have added the websites ranked
$1-200,1000-1100,10000-10100$, and 15000-15100 on alexa.com. We have eliminated any websites with fewer than five comments on Web of Trust. We have then sorted the remaining 456 websites by the human-provided scam score available on Web of Trust. We have used top 150 websites as negative and bottom 150 websites as positive instances of scam.

3. Spam emails: We have obtained a dataset of 1551 spam emails from a corporate email system, detected with high confidence by McAfee AntiSpam in November 2010. We have extracted 11825 web links from those emails. Since spam emails sometimes provide links to legitimate highranked websites to trick the spam filters, we have eliminated all links that are listed in the top one million websites on alexa.com. We have then identified the links that occurred at least ten times and used them as scam examples.

4. hpHosts: To test the effectiveness of our approach on new threats, we have taken the 100 most recent reported websites on the blacklist at hosts-file. net.

5. Top websites: This set is the top 100 websites according to the ranking on alexa.com. We have used them as examples of non-scam.

\begin{tabular}{l|c|c|c}
\hline Dataset Name & Scam & Non-scam & Total \\
\hline Scam Queries & 33 & 63 & 96 \\
Web of Trust & 150 & 150 & 300 \\
Spam Emails & 241 & none & 241 \\
hpHosts & 100 & none & 100 \\
Top Websites & none & 100 & 100 \\
All combined & $\mathbf{5 2 4}$ & $\mathbf{3 1 3}$ & $\mathbf{8 3 7}$ \\
\hline
\end{tabular}

Table 1. Labeled datasets for scam detection.

\section{Host ANALYZER Web SERVICE}

We have created a web service for collecting information about websites. This service is a collection of HTML wrappers, which are small programs that extract useful parts of web pages. We call each piece of collected information a feature. For example, traffic_rank is an integer feature obtained from alexa.com. We currently collect 43 features from 11 online sources (Table 2). We use these features as input to a supervised learning algorithm, which constructs a classifier for identifying spam pages. Most features are integers or real numbers, used as is. traffic rank and sites_linking_in features have larger order of magnitude and were replaced by the value of $\log ($ feature $)+1$. Booleans are converted to $\{0,1\}$. Nominal features, such as the country code, are converted to an integer which corresponds to the order they appeared first in the dataset.

The feature vector of a website, especially for less popular websites, may have many missing values. For example, to extract features from Wikipedia, we need to identify the company name corresponding to a website. If Host Analyzer cannot automatically find the company name, then all features obtained from Wikipedia are missing. 


\section{Approach}

We have used the logistic regression algorithm with L1 regularization [Mitchell, 1997; Schmidt et al., 2007]. We give a brief overview of this algorithm and explain its application to the scam detection.

We represent each dataset of websites as $D_{k}$ which is a set of feature vectors $x_{i}$.

$$
D_{k}=\left\{x_{i}=\left(1, x_{i 1}, x_{i 2}, \ldots, x_{i m}\right)\right\}_{i=1}^{n}
$$

In current version of the system, $m=43$ and $x_{i j}$ is the values of feature $j$.

Let $y_{i} \in\{-1$ (not scam), 1 (scam) $\}$ be the labels for the website represented by $x_{i}$. In logistic regression, the probability of $x_{i}$ being scam is:

$$
\begin{array}{r}
P\left(y_{i}=1 \mid x_{i}, w\right)=\frac{1}{1+\exp (w \cdot x)} \\
\text { where } \quad w \cdot x=\sum_{j} x_{i j} w_{j}
\end{array}
$$

The training procedure learns $w$ by maximizing the following convex $\log$-likelihood function with $\lambda$ being the regularization parameter and $\|w\|_{1}=\sum_{j}\left|w_{j}\right|$ is the L1-norm of the weight vector:

$$
l(w)=\sum_{i} \log \left(P\left(y_{i} \mid x_{i}, w\right)\right)-\lambda\|w\|_{1}
$$

This form of objective function has L1 regularization term $\lambda\|w\|_{1}$ and this negative term in the maximization problem, prevents the weights from getting too large and avoids overfitting. Optimization procedure with L1 regularization is different from the usual gradient ascent because $l(w)$ is not differentiable at points where weights are zero. We have used the projection approach by Schmidt et al. [2007] because of its scalability and fast convergence.

In addition to preventing overfitting, L1 regularization produces sparse weight vector $w$; that is, the resulting vector contains many zeros. The advantage of the sparse weight solution is that the features corresponding to zero weight values are not affecting the classification decision, which means that we can omit those features in our data collection.

A desirable property of logistic regression for our task is that the classifier outputs the probability of the website being scam (Equation 1). To make the prediction about a new website, we first need to create its feature vector $x_{\text {query }}$ using Host Analyzer, then we calculate $x_{\text {query }} \cdot w$ and compare it to a pre-set threshold:

$$
y_{\text {pred }}= \begin{cases}1 & x_{\text {query }} \cdot w \geq t \\ -1 & x_{\text {query }} \cdot w<t\end{cases}
$$

We have used the threshold of $t=0$ in the reported experiments which corresponds to $P\left(y_{i}=1 \mid x_{i}, w\right)=0.5$. That is, we consider the website as scam if it is more that $50 \%$ chance of being scam. The threshold can be adjusted to achieve the desired precision and recall trade-off.

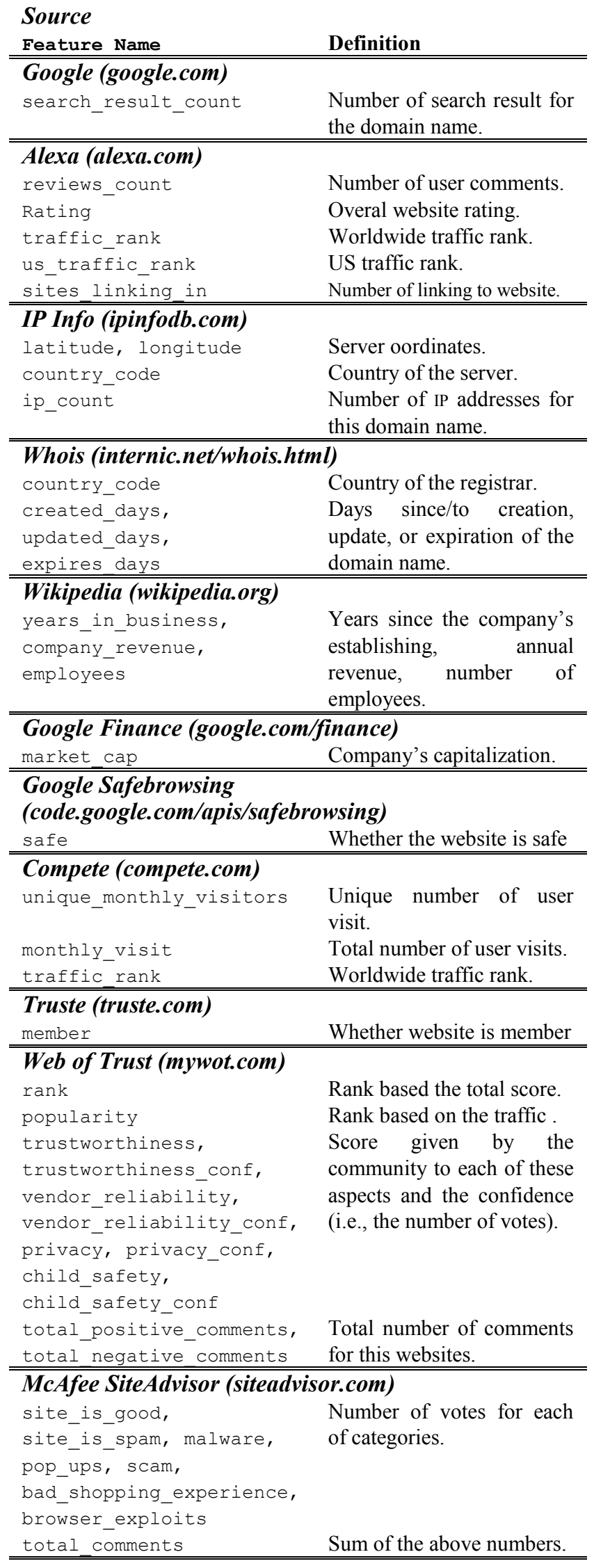

Table 2. Website features collected by Host Analyzer. 


\section{Experiments}

We have tested the developed technique using ten-fold crossvalidation on each dataset, and we summarize the results in Table 3. We have used the following four performance measures.

- Precision: The ratio of correctly predicted positives to the number of positive predictions.

- Recall: The ratio of correctly predicted positives to the number of positive labels.

- F1: The harmonic mean of the precision and the recall.

- AUC: The area under the ROC curve.

\begin{tabular}{|l|c|c|c|c|}
\hline Dataset Name & Precision & Recall & F1 & AUC \\
\hline Scam Queries & 0.9833 & 0.9667 & 0.9749 & 0.9667 \\
\hline Web of Trust & 0.9923 & 0.9923 & 0.9923 & 0.9990 \\
\hline Spam Email & 1.0000 & 0.9627 & 0.9809 & - \\
\hline hpHosts & 1.0000 & 0.9200 & 0.9583 & - \\
\hline Top Websites & 1.0000 & 0.9600 & 0.9795 & - \\
\hline All & $\mathbf{0 . 9 7 9 5}$ & $\mathbf{0 . 9 8 1 3}$ & $\mathbf{0 . 9 8 0 3}$ & $\mathbf{0 . 9 8 5 8}$ \\
\hline All w/o WOT & 0.9307 & 0.9773 & 0.9534 & 0.9582 \\
\hline
\end{tabular}

Table 2. Summary of scam detection results.

For the Web of Trust dataset, since we selected the labels based on the information from their website, we have removed 12 of the features which were collected from the same website. Even with fewer features the performance on this dataset was the best among all datasets. This can be explained by our selection criteria which was biased toward more popular websites; and also by the fact that websites with the Web of Trust scores have more information available from other sources.

Training cannot be done on the Spam Email, hpHosts, or Top Websites datasets because they have either only spam or only non-spam instances. The performance for those datasets is reported using a model trained on both Scam Queries and Web of Trust. Lastly, we combine all five datasets and report the results with and without the Web-of-Trust-related features.

Since we do not have any previous work to compare with our work, in Figure 1, we compare our results to three baselines: a trivial random classifier baseline and two strong features in our dataset, when used independently: the number of search results from Google and the traffic rank from Alexa. AUC performance is measured by treating the feature values as the classifier output and only for the instances where the feature values were available and ignoring the rest $(70 \%$ and $61 \%$ of the dataset respectively). We also provide a comparison between web scam detection and the state of art from the related tasks: email spam [Cormack, 2007a] and web spam detection [Cormack, 2010].

We need to choose regularization parameters $\lambda$ in Equation 1 , which controls the penalty of objective function for large weights. Higher values of $\lambda$ result in more zero feature weights in $w$.

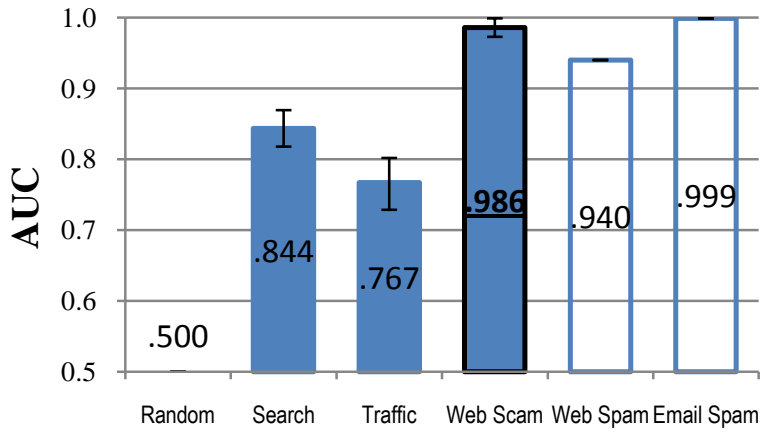

Figure 1. Comparison of detection performance to baselines and related tasks. Error bars are $95 \%$ confidence intervals.
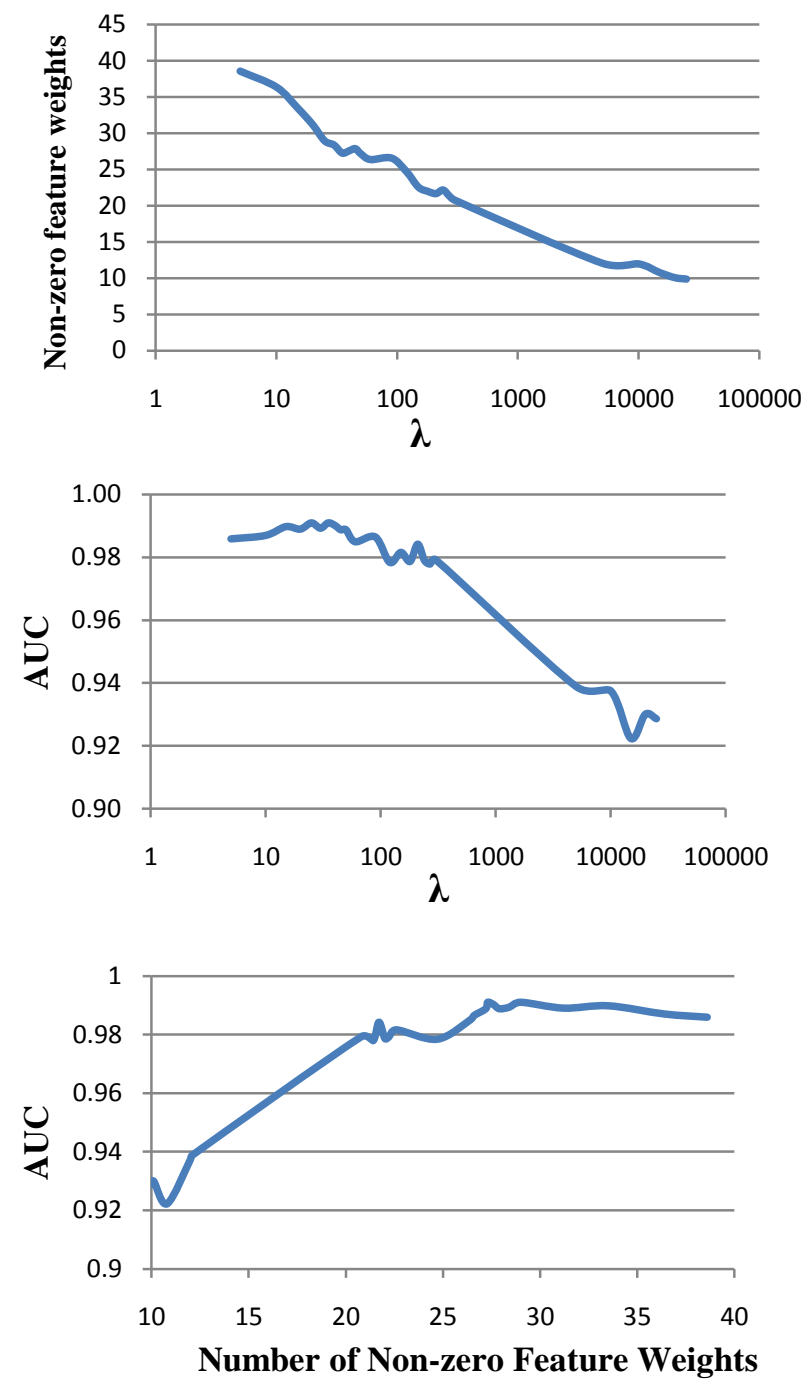

Figure 2 Effect of regularization parameter $\lambda$ on AUC and number of non-zero feature weights. Higher values of $\lambda$ result in fewer non-zero feature weights but reduce the accuracy.

Figure 2 shows the relation between $\lambda$, the number of non-zero feature weights, and the scam detection performance measured as AUC. We have set $\lambda=15$ for the results reported in Table 3 because as shown in 
Figure 2, the performance is relatively insensitive to the choice of this parameter for small values. We also show the larger values of $\lambda$, which pushes the model to be use fewer feature weights at some minimal performance cost. As mentioned previously, one advantage of sparsity in the model is the need for fewer features about the query website in the Host Analyzer.

The feature weights learned in logistic regression provide an insight about the importance of each feature. In Table 4, we show the top 10 positively weighted features and the top 10 negatively weighted features based the learned weight vector $w$ in Equation 1, along with the percentage of the dataset that has values for these features in the combined dataset.

\section{CONCLUSIONS AND FUTURE WORK}

We have presented work on detection of Internet scam based on automatically collected information about websites. The experiments show that both precision and recall of the developed technique is about $98 \%$. Informally, it means that the system correctly identifies scam in 49 out of 50 cases.

The current system is implemented as a Google Chrome browser extension, which is available for download at cyberpsa.com.

We plan to extend our datasets with better data collection process. Mechanical Turk data is noisy and human judgments approach may been improvement especially because these websites are optimized to trick user [Fogg et al., 2000].

\begin{tabular}{lrr} 
Negative-Weight Features & Weight & $\begin{array}{r}\text { \% with } \\
\text { value }\end{array}$ \\
\hline latitute & -0.2265 & $7 \%$ \\
site_is_good & -0.0494 & $32 \%$ \\
child_safety & -0.0314 & $58 \%$ \\
vendor_reliability & -0.0147 & $64 \%$ \\
total_positive_comments & -0.0144 & $35 \%$ \\
trustworthiness & -0.0087 & $65 \%$ \\
privacy & -0.0079 & $64 \%$ \\
total_negative_comments & -0.0011 & $48 \%$ \\
created_days & -0.0005 & $36 \%$ \\
employees & -0.0002 & $5 \%$ \\
& & \\
Positive-Weight Features & Weight & \% with \\
\hline traffic_rank & 0.2190 & $61 \%$ \\
search_result_count & 0.2092 & $70 \%$ \\
country_code & 0.0494 & $51 \%$ \\
malware & 0.0135 & $26 \%$ \\
updated_days & 0.0021 & $36 \%$ \\
expires_days & 0.0002 & $36 \%$ \\
ip_count & 0.0001 & $66 \%$ \\
scam & 0.0001 & $28 \%$ \\
reviews_count & 0.0001 & $26 \%$ \\
site_is_spam & 0.0001 & $29 \%$ \\
& &
\end{tabular}

Table 3. The top 10 positive and the top 10 negative feature weights. The right most column shows percentage of websites in the dataset for which Host Analyzer has automatically obtained the value of the related feature.

\section{REFERENCES}

[Anderson et al., 2007] Anderson, D. S., Fleizach, C., Savage, S., and Voelker, G. M. Spamscatter: Characterizing Internet scam hosting infrastructure. In Proceedings of the Sixteenth USENIX Security Symposium, 2007.

[Becchetti et al., 2008] Becchetti, L., Castillo, C., Donato, D., Baeza-Yates R., and Leonardi, S. Link analysis for web spam detection. ACM Transactions on the Web, 2(1):1-42, 2008.

[Cormack, 2007a] Cormack, G. V. TREC 2007 Spam track overview. In Proceedings of TREC 2007: The Sixteenth Text Retrieval Conference. 2007a.

[Cormack, 2007b] Cormack, G. V. Content-based Web spam detection. In Proceedings of the 3rd International Workshop on Adversarial Information Retrieval on the Web (AIRWeb), $2007 b$.

[Cormack, 2010] Cormack, G. V., Smucker, M. D., and Clarke, C. L. A. Efficient and Effective Spam Filtering and Re-ranking for Large Web Datasets. Unpublished Manuscript. 2010.

[Cova et al., 2010] Cova, M., Kruegel, C, and Vigna, G. Detection and analysis of drive-by-download attacks and malicious JavaScript code. In Proceedings of the Nineteenth International Conference on World Wide Web, pages 281290. 2010.

[Fogg et al., 2000] Fogg, B. J., Swani, P., Treinen, M., Marshall, J., Osipovich, A., Varma, C., Laraki, O., Fang, N., Paul, J., Rangnekar, A., \& Shon, J. Elements that affect web credibility: Early results from a self-report study. In $\mathrm{CHI}$ '00 extended abstracts on human factors in computing systems. 2000.

[Gyöngyi and Garcia-Molina, 2005] Gyöngyi, Z., and GarciaMolina, H. Web spam taxonomy. First International Workshop on Adversarial Information Retrieval on the Web, 2005.

[Kumaraguru et al., 2009] Kumaraguru, P., Cranshaw, J., Acquisti, A., Cranor, L., Hong, J., Blair, M. A., and Pham, T. School of phish: A real-world evaluation of anti-phishing training. In Proceedings of the Fifth Symposium on Usable Privacy and Security, pages 1-12, 2009.

[Mitchell, 1997] Mitchell, T. M. Machine learning. New York: McGraw-Hill, 1997.

[Schmidt et al., 2007] Schmidt, M., Fung, G., Rosales, R. Fast optimization methods for L1 regularization: a comparative study and two new approaches. In European Conference on Machine Learning (ECML), 2007.

[Sharifi et al. 2010] Sharifi, M., Fink, E., and Carbonell, J. G. Learning of personalized security settings. In Proceedings of the IEEE International Conference on Systems, Man, and Cybernetics, 2010. 